\title{
Biases in critical reading of TIS literature ${ }^{1}$
}

\author{
Daniel Gile*
}

\section{Introduction}

Educated laypersons know that scientists read relevant research literature in their discipline regularly so as to keep abreast of developments. Many are also aware of the peer review institution, which is supposed to ensure the high quality of publications. But how many are aware of the importance of the critical component in individual reading of scientific publications?

When reading the literature, researchers do not just seek information about new data, ideas and methods - they also assess them critically. Such assessments of the strengths and weaknesses of ideas in view of available data, of methods and of data collection and analysis, of inferences made on the basis of available data are a major driver of progress: critical reading raises their awareness of limitations of current knowledge, thinking and methods and helps them think of potentially promising avenues for correcting, strengthening and extending them.

The effectiveness of critical reading in detecting strengths and weaknesses in texts depends on several factors, one of which is obviously the critical reader's knowledge of the field, including the relevant literature, applicable scientific norms, availability, strengths and weaknesses of research methods. Without it, it is difficult to spot misrepresentations of theories and opinions, major omissions, sub-optimal choice and implementation of research methods. Which means that basically, newcomers to research will perform less well as critical readers than seasoned researchers, and especially seasoned researchers with

\footnotetext{
${ }^{1}$ This is an original paper, which was never published before.

* Université Paris Sobonne-Nouvelle
} 
experience in the particular paradigm and (sub)field addressed by the author of the text.

Beyond research expertise, critical reading in science is influenced by what I will call 'social bias' - besides personal bias, an ethical issue which will not be addressed here. Science is intellectual and technical, but also highly social, insofar as it is practiced by strongly communicative and highly competitive interaction of scientific communities and groups. Individually, scientists compete for positions, for grants, for recognition. As social groups, many of them adhere to competing 'paradigms', which, in the case of the social and human sciences, including Translation and Interpreting Studies (TIS), can refer to 'theories' or conceptual frameworks (Interpretive Theory, Skopos Theory, Relevance Theory, Effort Models, Descriptive Translation Studies etc.) on one hand, and to epistemological and methodological preferences (empirical vs. theoretical or hermeneutic, quantitative vs. qualitative, experimental vs. naturalistic etc.) on the other. Popper's view of science as progressing through a process whereby theories are proposed, empirically tested and amended or replaced when the data 'falsify' them (Popper 1959/1992) does not take this social influence on board. Thomas Kuhn's analysis (1962/1970) does. According to him, the (r)evolution of science is largely driven by power struggles between groups who hold power and support a certain paradigm and other clans with other groups who support a competing paradigm and work to strengthen it until they take over with the new paradigm.

It is reasonable to expect that members of the same paradigmatic group will not be totally neutral when looking at research conducted within their paradigm or compatible with it vs. research done within a competing paradigm. Their social bias can obviously influence the assessment of a study's or text's overall contribution. Those who support a particular paradigm or theory may not assign the same weight to studies or findings that are in line with them - or not. For instance, researchers who strongly support experimental research may well tend to systematically rate the contribution of naturalistic studies as lower than the contribution of experimental studies. This is unavoidable and not necessarily 
undesirable, as it puts some pressure on each 'clan' to improve the quality of its research production.

At a more local level, bias can also have subtler effects and perhaps explain some misunderstandings. A few examples from TIS will be listed here, from texts I have written or co-authored, for the simple reason that I know what I and the co-author had in mind and can therefore make claims that I would not be able to make on behalf of others.

\section{The Effort Models: a cognitive-only view of interpreting?}

In a critique of the Effort Models, Pym (2008) suggests that they are "presented" as independent of the social situation (p. 86) and that Gile's pooling together of errors and omissions "seems to imply that all omissions are to be seen on the same level of errors, i.e. as indicators of lesser quality" (p. 87). He then goes on to suggest that some omissions reflect socially situated tactical choices.

Actually, in Gile (1995), which he quotes, a whole chapter (chapter 2 ) is devoted to communication and quality in terms of socially situated action, loyalty and interests of various actors, and chapters 5 and 7 specifically mention risk analysis in translation and in interpreting respectively. In chapter 3, on fidelity, the analysis of the information content of informative statements suggests that some omissions are actually desirable, and chapter 7 , on coping tactics, refers to "maximizing the communication impact of the speech" and again, to the idea that "the interpreter serves communication and keeps in mind the interests of the participants in communication" (p. 202).

In other words, contrary to Pym's statements, the Effort Models are introduced in Gile (1995) as a cognitively focused analysis of action situated and determined by a social environment, including norms and risks. Pym's contribution is to give more salience to the risk analysis aspect - which is far from "hidden" in Gile's model, as suggested by the title of Pym's chapter.

Why did Pym disregard the available evidence (something which he later acknowledged in a personal email exchange) of the social situatedness of the Effort Models? I can only speculate that since his focus 
was social risk-analysis and that the local focus of the Effort Models is cognitive, he set his sights on the contrast and devoted little attention to the overall framework in which the Models were placed.

\section{The Tightrope Hypothesis: a quantified hypothesis?}

Kilian Seeber, an interpreter from Geneva, was trained in the experimental psychology paradigm, strongly adheres to it (personal communication) and has a strong interest in cognitive load. In his analysis of findings of an interesting experiment in which he used pupil dilation measurements as an indicator of cognitive load during simultaneous interpreting between "structurally different languages", he says that:

\footnotetext{
the local fluctuations of cognitive load reflected in the model seem to be of a magnitude that does not lend support to Gile's "tightrope hypothesis," according to which "most of the time, interpreters work near saturation level" (Gile 1999). In fact, even if we assume that the local (and thus relative) maximum load experienced during any of the four strategies represents the absolute maximum load, interpreters still work below saturation levels a considerable part of the time (Seeber, 2011, p. 197).
}

There are a number of loopholes in this rationale. One can perhaps be explained through a concrete situation which is familiar to all simultaneous interpreters: while in the booth, interpreters realize that they are lagging too far behind a speaker and will not be able to catch up, and therefore decide to omit part of the utterance to avoid being overloaded and missing something more important. Such a tactical decision is motivated by awareness of closeness to cognitive saturation and results in an omission, but will not be associated with maximum instant cognitive load as indicated by pupil dilation, precisely because the interpreter decided to avoid such saturation and keep attentional resources available. In other words, assuming that pupillometric measurements are a reliable indicator of cognitive load, they only measure cognitive load associated with the tasks the interpreter decided to take on, not necessarily cognitive load associated with full successful interpreting of an utterance. Another example is that of 'individual deficits' (Gile, 2009, p. 170), i.e. an insufficient 
amount of attentional resources directed at one Effort to complete the task in which it is engaged. For instance, an interpreter may devote too much attention to note-taking or to speech production and not enough to listening and analyzing the incoming speech, which results in incorrect or incomplete comprehension of the message and generates an error or omission. This is not necessarily associated with very high cognitive load resulting from engagement with note- or speech production as could be measured pupillometrically, but is related to the limited availability of attentional resources - if attentional resources far exceeded the requirements, enough could be attributed to each Effort to avoid such individual deficits.

One illustrative metaphor would a comparison with a company that manages several activities and has a budget (and an account) for each. The Tightrope Hypothesis would translate into the idea that the company tends to operate near 'budgetary saturation'. The Hypothesis does not say exactly how near. The difference between mean expenditure level and the existing budget could be a matter of thousands of euros, tens of thousands of euros or hundreds of thousands of euros. In some cases, an increase in a few thousand euros in expenditure could be enough to overrun the budget and place the relevant account in the red, and in others, only much larger sums would significantly affect it. Moreover, knowing its budgetary limitations, the management may consider certain investments that would be required to optimize the operation of the company, decide they would be too expensive in view of budgetary limitations and give up on the idea. In such a case, failing to engage in these investments because of nearness to budgetary saturation would not be visible if the sole indicator of 'budgetary load' were measurement of effective expenditures versus the existing balance on the respective accounts.

Pupillometric indicators could perhaps be made more powerful experimentally, for instance by asking interpreters specifically to do their best to comprehensively interpret difficult utterances and observing pupillometric behavior before, during and right after breakdowns manifest through errors and omissions. But until this or similar studies are done, it is 
difficult to use quantitative pupillometric data to determine whether the Tightrope Hypothesis is correct or not.

Why did Seeber miss these points? I can only speculate that his paradigmatic bias - plus the fact that my own descriptions and discussions of the Models repeatedly refer to cognition - guided him toward an experimental, quantitative method with a somewhat excessively narrow focus.

\section{Lexical gaps in signed language interpreting: a sensitive issue}

Sophie Pointurier-Pournin, a French signed language interpreter and head of the signed language interpreting section at ESIT, Paris, wrote an MA thesis on interpreting tactics used by signed language interpreters working from French into French Sign Language (FSL) when encountering lexical gaps (Pointurier-Pournin, 2009). When she submitted a (co-authored) paper on the same topic to a journal, anonymous reviewers objected strongly to the focus on and concept of lexical gaps in the study, claiming that they suggested that French Signed Language was 'inferior' to French (the spoken language). As Pointurier-Pournin's MA and PhD supervisor, having discussed her work extensively with her, I could not detect any such idea in her interest and investigations. The text (Pointurier-Pournin and Gile, 2012) described and analyzed how interpreters coped with the lack of lexicalized signs in FSL for certain ideas expressed in single lexical units in French, the focus being on cognitive implications within the framework of the Effort Models.

The salience of lexical gaps in spoken language-into-signed language interpreting which stems from the difference in lexicon size between spoken languages and signed languages is clear to all signed language interpreters (see for instance Swabey et al., 2016). Why did reviewers object to its selection as an object of study and read value judgment into the manuscript? One possible reason is social bias. In France, there are basically two opposing 'academic clans' in the world of sign languages and signed language interpreting. In a nutshell, one takes a linguistic and strong pro-Deaf advocacy position, and the other takes a more neutral communications-oriented, Interpretive theory-based position. 
Pointurier-Pournin's manuscript was written in French, and judging by the comments received - in French - the reviewers were most probably from the opposite French clan, which could explain their (mistaken) perception of hidden suggestions in the text.

Does the peer review system, with generally involves at least two referees and one editor, provide immunity against such bias and make it possible to distinguish reliably strengths and weaknesses by combining and balancing against each other the result of each reader's scrutiny of submissions? It does, to some extent, provided the reviewers and editor(s) are not from the same paradigmatic or social 'clan' - but not if they are, and not if one of them is particularly vocal or has a particularly large weight associated with a solid reputation or institutional power. However, even in the absence of such imbalance between the respective influences of referees, the system is far from foolproof for another reason: critical reading is a rather intensive effort, is mostly done without any remuneration and competes with other scholarly and other activities, and the time and attention devoted to it by individual readers are generally not sufficient to detect all strengths and weaknesses potentially detectable by them on the basis of their knowledge and expertise. Over the years, I have been struck time and again, when reading other peer reviewer's reports of texts which I had also refereed, how much could be missed by individual readers, including myself. Actually, during vivas of my own students' masters' theses and doctoral dissertations, which I read and discuss with them repeatedly, the comments of other members of the defense committee regularly demonstrate that despite my best efforts, I often miss some of their weaknesses.

Under the assumption of the referees' honesty, imperfections in the product of critical reading can thus result from a combination of relevant expertise gaps, social bias and insufficient attention in individual readers which are not necessarily fully compensated by the peer review system. Some authors even claim that the peer review system is basically flawed (Smith, 2006), though I am not aware of anyone suggesting that it does not contribute anything and should be dispensed with. 


\section{'Objective flaws' or 'subjective dislikes'?}

When reading literary works, readers occasionally encounter typing errors, language errors and factual errors. These are prototypical 'objective flaws', insofar as any person with sufficient knowledge of the relevant language, including the author, would agree that they are errors. But readers may also criticize a plot, a style, a description because they do not like them, while others do. Such dislikes express subjective preferences (or rather unpreferences), but cannot be taken as indicators of 'objective flaws' as long as the incriminated features of the text are not in contradiction with a wellestablished linguistic or literary norm which is clearly applicable to it.

When critically reading scientific texts, a similar categorization is useful. Typical 'objective flaws' include typing errors, calculation errors, factual errors (for instance as regards volume, mass, speed, colors, frequency of occurrence, population size, known behavior patterns). If these are pointed out with - in the case of factual errors - reliable evidence (i.e. considered as such in the relevant scientific community), there should be no disagreement as to their being errors. Typical dislikes relate to the choice of bibliographical references, to editorial features, to non-standard lexical and terminological choices, and even to the choice of research topics and research questions, which some critical readers may consider interesting and/or useful and other irrelevant or futile. There are also stylistic preferences, some of which are discipline-specific or paradigmspecific, and some are associated with culture in the wide sense. For instance, in an interesting doctoral dissertation, Karen Bennett (2008) discusses stylistic preferences in Portuguese (flowery) vs. Anglo-saxon (simple and economical) scientific writing.

Between the two poles, definitions of what is a flaw and what is a dislike can be analyzed sociologically as governed by social norms at different levels. Following Snow (1990), I have suggested elsewhere (see for instance Gile, 2013) that it makes sense to think of 'science', as it is now recognized institutionally, as made up of two distinct scientific cultures, or rather two distinct families of scientific culture. The first, which I call 'canonical science culture' (CSC), is intrinsically empirical and views evidence in the form of data and cautious logical reasoning as fundamental 
for exploration of reality and validation of ideas. The second will be referred to here, somewhat unsatisfactorily, as the 'human sciences culture' (HSC), because it is typical of many - albeit not all - human sciences. Theoretical analysis and discussion of other scholars' ideas rather than data from the field or the laboratory are its main engine for progress, and it is much bolder than CSC in constructing and adopting theories and in assigning 'meaning' to phenomena with available evidence. Also, in HSC, interpreting statements and claims as reflecting hidden meaning and agendas is not necessarily frowned upon, whereas it generally runs against fundamental norms in CSC.

For instance, when analyzing a text, on the basis of personal experience and knowledge, an HSC scholar might point to the use of some nouns, verbs and adjectives and interpret them as reflecting an author's political or ideological position without further ado. Typically, an CSC researcher would rather calculate how frequently they occur in this text and in other texts known to be associated with similar and other political or ideological positions and write that such evidence as presented in his/her report is consistent (or not) with the hypothesis that the text's author takes this or that particular position.

Within each of these two large families, operational research norms can differ markedly. For instance, being rigorous about collecting and analyzing data does not translate into the same rules in experimental physics and in ethnological research (which is classified as a social or human science, but can still be viewed as part of the CSC family) or in historical research (which is sometimes very much within a CSC paradigm and sometimes closer to HSC). Even within psychology, social psychology and cognitive psychology both use experiments, but with different designs and metrics (for instance Likert scale values for the former and reaction times and proportions of errors for the latter).

Such methods and norms reflect research traditions as well as adaptation to the object of investigation, to environmental constraints and to available techniques and technology. An approach germane to one particular research question and to a particular environment, for instance experimental design with physiological measurements and strict control of 
potential confounds, can be virtually useless if not unfeasible when attempting to answer another research question or the same research question in another environment, if for instance it is not possible to construct sufficiently large representative samples. And yet, researchers who have been trained and have worked throughout their career within a certain tradition can be reluctant to acknowledge that other methods and other norms may be 'better' in some environments. In TIS, I have often (but not always) felt such reluctance in colleagues who were trained in the experimental paradigm of cognitive psychology.

With respect to the difference between 'objective flaws' and 'subjective dislikes, it follows from this analysis that the boundaries depend inter alia on the academic territory: just as some acts are legal in one country and illegal in another, what is considered a flaw in one academic community can be viewed as a mere dislike - or perfectly acceptable in another.

\section{Limitations vs. flaws}

Another useful distinction in the context of critical reading is that between limitations and flaws. A flaw is an error, or clear sub-optimal use of available resources. A limitation involves no errors, but is due to constraints and/or lack of resources.

For instance, as is often mentioned in the literature, it is difficult to enlist professional interpreters as participants for experimental studies. As a result, most experiments on professional conference interpreting, say on note-taking in consecutive, rely on small samples, which turns them into case studies for all intents and purposes. The resulting lack of generalizability of findings of individual studies is a limitation rather than a flaw, because it is due not to an error or sub-optimal use of resources, but to constraints associated with the lack of resources. Enlisting interpreting students to obtain larger samples would be a flaw insofar as it is believed that mastering note-taking skills takes much time, and the way professionals with some experience go about it is likely to differ markedly from the way students go about it. 
In one research project on directionality with English and French, the researcher only had access to four interpreters, two with an English A and a French B and the other two with a French A and an English B. The researcher decided to have all of them interpret an English speech into French, which made it possible to compare two A-into-B performances with two B-into-A performances. Had she given them two speeches, one in French to interpret into English, and one in English to interpret into French, she could have had a total of 8 interpreting performances to compare, four into $A$ and four into $B$, and every interpreter would have had to work into $A$ and into $B$, a design which would have been potentially more powerful for comparisons. While the main constraint was limited availability of interpreter participants, speeches can be found or prepared easily. In this case, there was a flaw in the design, as resources were used sub-optimally.

\section{Practitioner bias}

One interesting feature of the interpreting research community is that it is overwhelmingly populated by 'practisearchers', practitioners of interpreting who also engage in research, most of whom (but this is changing) have received little or no systematic training in research methods. Depending on the school of thought within which they started doing research (Interpretive Theory in Paris, Skopos Theory in many German speaking and Nordic countries, cognitive psychology in Geneva, corpus linguistics in Forlì) and on the strength of assimilation of the research paradigms they studied, they sometimes have a rather limited understanding of the rationale underlying the use of techniques and methods and do not understand the mismatch between some of them and the relevant interpreting environment being investigated. Among these practisearchers, some have inherited the strong bias of their predecessors (e.g. Seleskovitch and Lederer and their followers in the 1970s and 1980s) against research procedures that do not investigate actual interpreting in a natural environment.

Many objections voiced against early experiments that used noninterpreters and non-interpreting tasks to investigate interpreting are justified, but systematic rejection of studies with interpreter participants 
and non-interpreting tasks is not desirable and deprives the community of some potential inroads into better comprehension of the interpreters' lexicon, bilingualism and cognition, which are relevant to interpreting as such in ways which remain to be fully explored.

The particular demographics and backgrounds found within the interpreting research community are thus associated with what could be called 'practitioner bias', as distinct from bias induced by academic norms assimilated during early research training in other disciplines.

\section{Reducing the undesirable effects of social bias in critical reading}

It was suggested earlier that negative bias was not always undesirable. It raises awareness of weaknesses in texts and can generate feedback that can help authors improve their scholarship. It is harmful when it causes authors to be treated unfairly by ignoring merits in their texts and by misrepresenting their views and work.

Paradigmatic bias, which is associated with a general preference within one scientific community for some norms over others which are preferred in another community, may result in damage to individual researchers and studies through negative assessments by peer reviewers, but cannot be considered 'unfair'. To prevent such damage, the most straightforward way is to publish within the scientific community whose norms the author follows. In some cases, such norms are innovative or are not accepted by the author's home community, and there may be a lot of convincing to do before they gain acceptance.

In TIS, where research communities take inspiration from different research communities with different norms (experimental psychologists, ethnologists, corpus linguists etc.), young researchers can be made aware by their trainers and supervisors of the possibility and effects of social bias associated with such differences so that they can understand some criticism that might be leveled at them. In order for their own critical reading to be as productive and free of such bias as possible, it is best if they are also introduced to various research paradigms and made to appreciate the limitations and advantages of each for various research questions and in various environments. 
Providing full research training to interpreting students or even graduate/doctoral students in several research paradigms is not feasible nor desirable, because it would take too long. Breaking or at least mitigating misconceptions can probably be achieved more easily through a guided tour of selected parts of the history of science. For instance, it is easy to show through readings in the history of astronomy, zoology, geology, linguistics, epidemiology and through scientific extension articles that 'good science' does not necessarily rely on experiments or on quantitative research, and through readings in the history of arts that empirical research can be found even in typical human sciences. This should provide them with some immunity against pre-conceived ideas about what 'good science' is in terms of research methods (I recently received from a well-read colleague with interest in research a mail in which he suggested that "real science" [necessarily] involved controlled experiments), sample sizes or inferential statistics and perhaps leave their mind more open to assessments based on the following criterion: "is the method used in this study appropriate in view of the research question asked, existing constraints and available resources?"

\section{Practical training}

As is the case of many if not all skills, critical reading is probably best learned through guided hands-on exercises. Awareness raising and critical reading workshops in two to three half-day sessions set about a week apart can be a good starting point:

1. Awareness can be raised in two phases, with discussions and exercises moderated by informed trainers with a good understanding of the issues involved, an open mind and willingness to take some distance from their own positions and look at them self-critically:

- A general introduction to critical reading in science (for instance something along the lines of Gile, 2001)

- A discussion by a trainer of published book reviews which provide different assessments of the same book, or of book reviews with which s/he does not agree, the idea being to detect and discuss actual flaws 
if any in the reviews as well as differences in the likes/dislikes of the authors of the reviews and of the trainer.

\section{Practical critical reading workshops:}

Participants are assigned texts reporting empirical studies (see explanations below) with the task of reading them critically over a few days and preparing written reports which will be discussed in class.

In order to ensure that they actually read the texts for overall comprehension, their reports should include a short but informative summary of the studies (preferably less than 200 words) with a (re)formulation of the research question(s), a synopsis of the type of research design, the materials and methods, the results and the author's conclusion(s).

In a second part, participants should analyze the studies' strengths and weaknesses as regards substance (literature review, design of the study, implementation, inferences), and in a third part, strengths and weaknesses of form are listed separately (overall structure of the text, layout, language, tables and graphs, bibliographical norms).

Critical reading is part of researcher's scientific activity and should be as systematic and careful as research projects. One good way of going about it as a beginner (and beyond) is to read the relevant text line by line and annotate it as it is being read, using the comment insertion function, writing in the margins etc., depending on the format of the text, the relevant medium (hard copy or electronic file) and available writing tools. Electronic formats are convenient for this purpose, and even pdf files can be annotated (highlighted and commented) with suitable inexpensive software.

Ideally, a text reporting an empirical study should take readers by the hand and gradually introduce them to the study, starting with an introduction, which includes a literature review leading up to the purpose and research question(s) which will be addressed in the study; a presentation of materials and methods follows, then a presentation and discussion of results, then a conclusion, references and annexes if any. Critical readers can therefore check line by line, as they read the text, 
whether everything is clear, whether the information provided is correct and relevant, whether they agree with the author's strategies and decisions, whether inferences made are justified by the available data.

Once the whole text has been read and annotated, a self-critical run through the comments should be an opportunity to re-assess and correct them if necessary. Are they formulated clearly enough? Are they justified? Perhaps a comment made at one point while reading the text on the absence of some information or bibliographical reference has become obsolete because the information or reference was provided elsewhere. And perhaps some negative criticism, for instance on a sample being too small, or on the absence of inferential statistics in the analysis of the data, or on non-randomization of the allocation of members to groups which will be compared (such randomization is standard in controlled experiments to prevent bias), needs to be qualified or at least categorized as a limitation rather than as a flaw once environmental constraints have been taken on board.

At the next step, a short synopsis of the content can be written, as well as the two reviews, one on content and one on form, taking on board all the annotations, again in a self-critical mind, trying to detect any bias in one's negative criticism.

An important moment in the training process is the discussion of the critical reviews in class. As suggested above, this discussion should be led by an open-minded trainer with experience in more than one scientific paradigm. An alternative solution is the participation of two trainers from different scientific paradigms, the idea being that convergences and divergences between them can be instructive about the existence of different norms in science. In both cases, the trainer(s)/moderator(s) can proceed systematically with a paragraph-by-paragraph critical reading of the text assigned to the participants, asking them for their comments, both individually and collectively. The ensuing discussion in class can highlight the relative nature of preferences versus consensus on 'real flaws', as well as differences between flaws and limitations - a crucial question when a comment is made on an alleged flaw in a study is whether the author of the criticism can suggest a better way to proceed with existing resources. In 
some cases, it should have been clear from the start that the resources were too scarce/the constraints were too heavy for a meaningful attempt to answering the particular research question addressed. Alternative research questions could have made the research project feasible, and the moderator(s) can guide the participants towards ideas about such alternative research questions.

The exercises should be repeated with at least two, perhaps three texts, which should be selected so that the language and methodology are relatively easy to understand even for beginners.

As mentioned earlier, this type of exercise is best done with texts reporting empirical studies within CSC (which need not be quantitative), not because of any alleged 'superiority' of empirical research, but because it is easier in such texts, in which all inferences are supposed to be based on explicitly presented data sets, to identify flaws and to discuss preferences. In particular, in CSC as opposed to HSC, no hidden meanings and suggested implications of particular statements and choices, ideological or otherwise, are assumed or discussed. Again, there is no denying the potential importance of such hidden meanings and ideological biases and motives in research choices and action. Just as a photograph is framed in a certain way, perhaps with a certain form of lighting and therefore reflects and highlights some aspects of reality while toning down or excluding other aspects, perhaps deliberately, perhaps for an affective or ideological reason. But the analysis of such hidden dimensions of research is hazardous, as illustrated in the discussion between Pöchhacker and Gile in Schäffner (2004). Before engaging in such analysis, as in Critical Discourse Analysis, it is perhaps better to acquire rigorous critical reading skills based on explicitly presented data and becoming aware of the pitfalls of bias.

\section{An ultimate step in favor of fairness}

Awareness of the existence and potential effects of critical reader bias is an asset, perhaps a strong one, which is likely to prevent some unjustified criticism and associated damage and perhaps to improve the quality of assessments. However, it is not a universal, all-powerful remedy. In two specific cases, it is often possible to go one step further: in peer reviews, 
when authors of peer-reviewed manuscripts are given the chance to read and react to comments made by assessors, provided their responses are given due consideration by the editors even if they are inexperienced and the peer reviewers are well-known; and in the case of book reviews to be published in a journal, when the reviewer sends a draft review to the author(s)/editor(s) and asks whether anything of some importance in the book has been missed, misunderstood or misrepresented. In my experience, authors/editors have always appreciated being informed and consulted, and they have never applied any pressure to obtain a change in my assessment.

Science is sometimes said to be a series of approximate accounts of reality which improve over time. By honing our critical reading skills, in particular as regards bias prevention, we can help it progress more efficiently, especially in a discipline such as TIS where research training is not yet fully developed.

\section{References}

BENNETT, K. English academic discourse: its hegemonic status and implications for translation. Doctoral dissertation, University of Lisbon, 2008.

GILE, D. Basic Concepts and Models for Interpreter and Translator Training. Amsterdam/Philadelphia: John Benjamins, 1995/2009. Critical reading in (interpretation) research. In Gile, Daniel; Dam, Helle; Dubslaff, Friedel; Martinsen, Bodil; Scholdager, Anne (eds). Getting Started in Interpreting Research. Amsterdam/Philadelphia: John Benjamins, 2001, p. 23-38.

Scientificity and Theory in Translation Studies. In Gambier, Yves \& Luc Van Doorslaer (eds). Handbook of Translation Studies, Volume 4, p. 148-155. Amsterdam/Philadelphia: John Benjamins, 2013.

KUHN, T. The structure of scientific revolutions. Chicago: Chicago University Press, 1962/1970. 
POINTURIER-POURNIN, S. L'interprétation du vide lexical des domaines de spécialité. Une étude de cas. Unpublished MA thesis, ESIT, Université Paris 3 Sorbonne Nouvelle, 2009.

POINTURIER-POURNIN, S., GILE, D. Les tactiques de l'interprète en langue des signes face au vide lexical: une étude de cas. Jostrans $\mathrm{n}^{\circ} 17, \mathrm{p}$. 164-183, 2012.

POPPER, K. The logic of scientific discovery. London and New York: Routledge, 1959/1992.

PYM, A. On omission in simultaneous interpreting. Risk analysis of a hidden effort. In Hansen, Gyde; Chesterman, Andrew; GerzymischArbogast, Heidrun (eds). Efforts and Models in Interpreting and Translation Research. Amsterdam/Philadelphia: John Benjamins, 2008, p. 83-105.

SCHÄFFNER, C. (ed.). Translation Research and Interpreting Research. Traditions, Gaps and Synergies. Clevedon, Buffalo, Toronto: Multilingual Matters, 2004.

SMITH, R. Peer review: a flawed process at the heart of science and journals. Journal of the Royal Society of Medicine 99, p. 179-182, 2006.

SNOW, C.P. The two cultures. Leonardo 23:2-3, p. 169-173, 1990.

SWABEY, L.; NICODEMUS, B.; TAYLOR, M.; GILE, D. Lexical decisions and related cognitive issues in spoken and signed language interpreting: $\mathrm{A}$ case study of Obama's inaugural address. Interpreting 18:1, p. 34-56, 2016.

\footnotetext{
Abstract

Critical reading is presented as a research skill, and the existence and potential effects of 'social bias', including practitioner bias, are explained. Against this background, a distinction is made between 'objective flaws' and 'subjective dislikes', and between flaws and limitations. After a reminder of some particular features of Translation and Interpreting Studies authors, practical training in critical reading with a focus on the reduction of undesirable effects of bias is outlined, and steps in peer reviewing and book reviewing allowing authors to react to criticism are recommended.
} 
Keywords: Critical reading; social bias; flaws vs. dislikes; researcher training; peer reviews

\section{Resumo}

A leitura crítica é apresentada como uma habilidade em pesquisa, e são explicados a existência e os potenciais efeitos do "viés social", incluindo o viés do profissional. Nesse contexto, faz-se uma distinção entre "falhas objetivas" e "desagrados subjetivos", bem como entre falhas e limitações. Após uma recapitulação de algumas características específicas de autores dos Estudos da Tradução e da Interpretação, é delineada a formação prática em leitura crítica com foco na redução dos efeitos indesejados do viés e são recomendados alguns passos para revisão entre pares e crítica de livros que permitam aos autores reagir a eventuais críticas.

Palavras-chave: Leitura crítica; viés social; falhas vs. desagrados; formação de pesquisadores; revisão entre pares 